Harald Mehlich

Electronic Government 
Harald Mehlich

\section{Electronic Government}

Die elektronische Verwaltungsreform

Grundlagen - Entwicklungsstand -

Zukunftsperspektiven 
Bibliografische Information Der Deutschen Bibliothek

Die Deutsche Bibliothek verzeichnet diese Publikation in der Deutschen Nationalbibliografie; detaillierte bibliografische Daten sind im Internet über <http://dnb.ddb.de> abrufbar.

Dr. Harald Mehlich ist Professor an der Hochschule für öffentliche Verwaltung und Finanzen in Ludwigsburg. Er lehrt Verwaltungsinformatik und Planen und Entscheiden.

1. Auflage Oktober 2002

Alle Rechte vorbehalten

(C) Betriebswirtschaftlicher Verlag Dr. Th. Gabler $\mathrm{GmbH}$, Wiesbaden 2002

Lektorat: Karin Janssen

Der Gabler Verlag ist ein Unternehmen der Fachverlagsgruppe BertelsmannSpringer. www.gabler.de

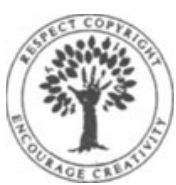

Das Werk einschließlich aller seiner Teile ist urheberrechtlich geschützt. Jede Verwertung außerhalb der engen Grenzen des Urheberrechtsgesetzes ist ohne Zustimmung des Verlages unzulässig und strafbar. Das gilt insbesondere für Vervielfältigungen, Übersetzungen, Mikroverfilmungen und die Einspeicherung und Verarbeitung in elektronischen Systemen.

Die Wiedergabe von Gebrauchsnamen, Handelsnamen, Warenbezeichnungen usw. in diesem Werk berechtigt auch ohne besondere Kennzeichnung nicht zu der Annahme, dass solche Namen im Sinne der Warenzeichen- und Markenschutz-Gesetzgebung als frei zu betrachten wären und daher von jedermann benutzt werden dürtten.

Umschlaggestaltung: Ulrike Weigel, www.CorporateDesignGroup.de

Gedruckt auf säurefreiem und chlorfrei gebleichtem Papier

ISBN-13:978-3-409-12295-5 e-ISBN-13:978-3-322-87000-1

DOI: $10.1007 / 978-3-322-87000-1$ 


\section{Vorwort}

Mit dem beginnenden Electronic Government (eGovernment) geht eine erste Ära des EDV-Einsatzes in der öffentlichen Verwaltung zu Ende. Wurde die EDV traditionell nur für begrenzte Zwecke wie $z$. B. Einwohnermeldewesen eingesetzt und ausschließlich von den Verwaltungsmitarbeitern bedient, so zeichnet sich mit dem eGovernment eine gänzlich neuartige Verwaltung und demokratische Praxis ab. Erstmalig verfügen die Bürger über einen direkten Zugriff auf die Verwaltungsdienstleistungen über das Internet. Durch den schnelleren und komfortablen Zugang läßt sich die Kunden- und Serviceorientierung entscheidend steigern. Bei einfachen Dienstleistungen ist dies schon heute möglich.

Bürgerfreundlichkeit läßt sich auf einem neuartigen Niveau umsetzen, wird jedoch bis zu einer flächendeckenden Umsetzung noch einige Zeit auf sich warten lassen: mit wenigen MausClicks sind dann beispielsweise sämtliche Verwaltungsformalitäten im Zusammenhang mit einem Wohnsitzwechsel erledigt. Mit der heute verfügbaren Technik ist die Idee eines One-Stop-Governments und einer Inanspruchnahme von Verwaltungsleistungen durch Online-Selbstbedienung erstmals in greifbare Nähe gerückt.

Die flächendeckende Vernetzung durch das Internet ist eine Schlüsselvoraussetzung für eGovernment. Damit wird eine umfassende Vernetzung von Prozessen innerhalb einer Behörde sowie zwischen den einzelnen Verwaltungen ermöglicht.

eGovernment steht für den Umbau der bisherigen „Offline-Verwaltung“ zu einer durchweg online zugänglichen Verwaltung. Mit dem eGovernment zeichnet sich erstmalig eine Verwaltungsreform auf elektronischer Grundlage ab.

Dahinter verbirgt sich mittlerweile eine kaum noch überschaubare Zahl von Projekten, Konzepten und Initiativen auf verschiedenen Verwaltungs- und Politikebenen. Gemessen an den hochgesteckten Zielen vollzog sich der Übergang zur Online-Verwaltung bisher schleppend.

Inzwischen ist deutlich geworden, daß die infrastrukturellen und rechtlichen Voraussetzungen weithin fehlen, um fortgeschrittene eGovernment-Lösungen umzusetzen. Dies überrascht wenig, weil nahezu der gesamte vorhandene rechtliche Rahmen noch immer auf die Funktionserfordernisse einer offline praktizierten Verwaltung ausgerichtet ist. Unterdessen sind die erforderlichen Anpassungsprozesse auf breiter Grundlage ins Rollen gekommen.

Das Buch vermittelt einen umfassenden Überblick über das mittlerweile weit verzweigte Themengebiet des eGovernments und stellt den aktuellen Entwicklungsstand, Erklärungskonzepte, Rahmenbedingungen und künftige Entwicklungsperspektiven dar.

An eine überblicksartige Einführung in das Themengebiet eGovernment (Kap. 1) schließt sich eine Abgrenzung gegenüber dem Verwaltungsreformansatz der neuen Steuerungsmodelle (Kap. 2) an. Es folgt eine Skizzierung vorliegender Erklärungsmodelle sowie eine Darstellung rechtlicher, organisatorischer, politischer und weiterer Rahmenbedingungen (Kap. 3-4). Der Anwendungsbezug wird hinsichtlich der Kommunikation zwischen der Verwaltung und ihren Kunden, der Intensität dieser Kommunikation sowie hin- 
sichtlich zentraler Strukturen im Front- und im Back-Office erläutert (Kap. 5). Der aktuelle Übergang zu fortgeschrittenen eGovernment-Lösungen ist höchst voraussetzungsvoll: Standardisierung, Integration im Back-Office und Infrastrukturerfordernisse wie die elektronische Signatur werden eingehend analysiert (Kap. 6). Die Veranschaulichung des aktuellen eGovernment-Entwicklungsstandes geschieht anhand konkreter Projektbeispiele (Kap. 7). Daran schließt sich eine Herausarbeitung künftiger Entwicklungsperspektiven unter dem Blickwinkel virtueller Verwaltungsstrukturen und möglicher Folgen für traditionelle Verwaltungsstrukturen an. Die hohe praktische Bedeutung des eGovernments kommt bei einem abschließenden Blick auf Benchmarking-Ansätze und konkrete Einstiegsstrategien der Verwaltungen zum Ausdruck.

Das Buch wendet sich an Interessenten aus der angewandten Informatik und aus den Verwaltungs- und Wirtschaftswissenschaften. Studierenden und Wissenschaftlern sowie Entscheidungsträgern und Praktikern aus Verwaltungen und öffentlichen Betrieben dient es als Einstieg und Übersicht in das Themengebiet.

An dieser Stelle danke ich meiner Familie und allen, die mich bei meiner Arbeit tatkräftig unterstützt haben.

Hinweise und Anregungen nehme ich unter harald.mehlich@t-online.de dankbar entgegen. 


\section{Inhaltsverzeichnis}

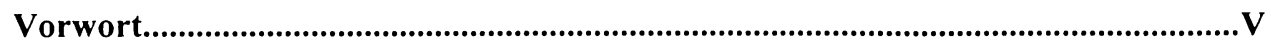

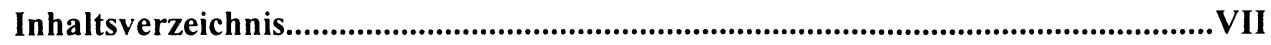

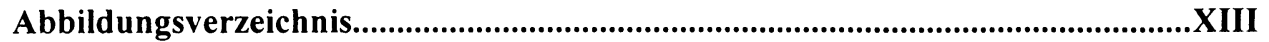

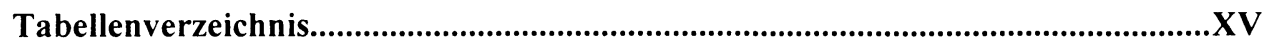

Abkürzungsverzeichnis...........................................................................................

1. EINLEITUNG ...........................................................................

$1.1 \quad$ Begriff eGovernment .....................................................................................

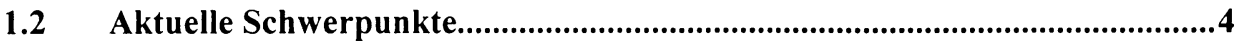

1.3 Verhältnis zur traditionellen Bürokratie ......................................................6

1.4 Institutionalisierung einer Leitreform ......................................................8

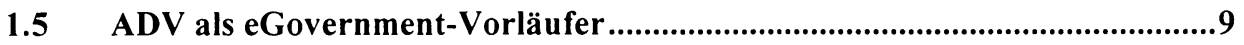

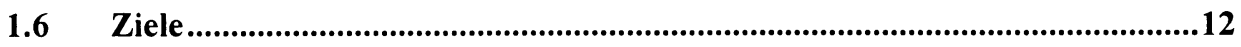

2. eGOVERNMENT UND NEW PUBLIC MANAGEMENT ........................ 15

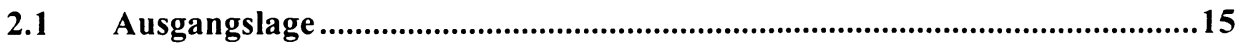

2.2 Kernelemente neuer Steuerungsmodelle .........................................................16

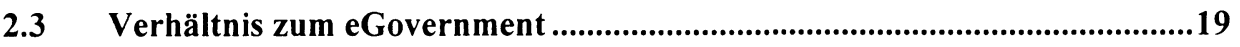

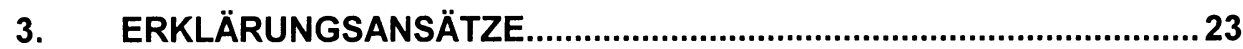

3.1 Typologien, Schichtenmodelle, Architekturen ............................................23

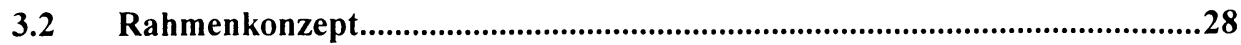

4. BASISTECHNOLOGIEN UND RAHMENBEDINGUNGEN ...................33

4.1 Informations- und kommunikationstechnologische Basisvoraussetzungen 33 
4.2 Rechtliche Rahmenbedingungen ....................................................................38

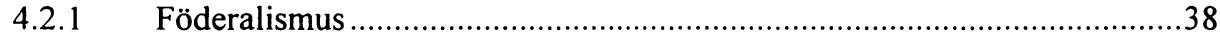

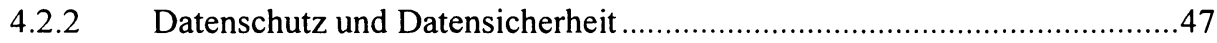

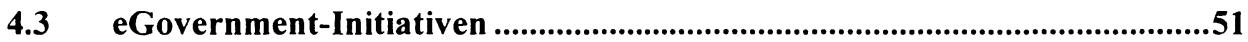

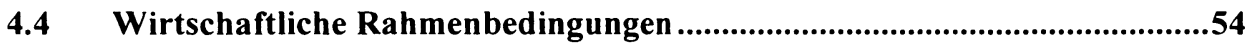

4.5 Soziale Akzeptanz und technischer Ausstattungsgrad ..............................55

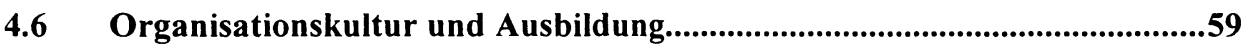

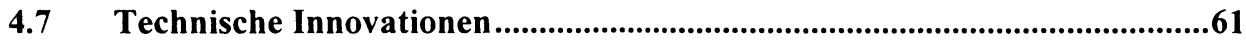

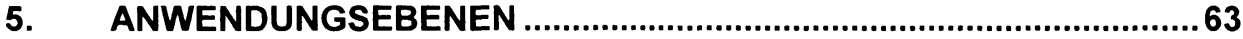

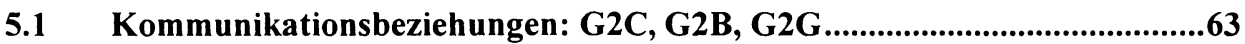

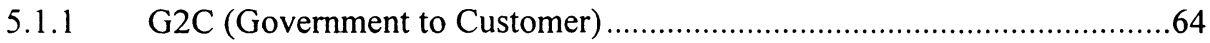

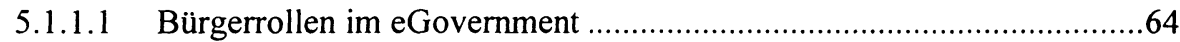

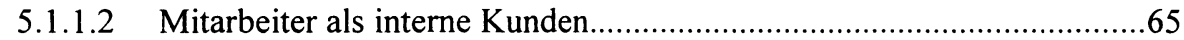

5.1.1.3 „Government“ im eGovernment........................................................66

5.1.1.4 Einfache und komplexe G2C-Beziehungen .....................................67

5.1.1.5 G2C-Beziehungskomplexität ...................................................68

5.1.1.6 Anonymität und geschlossene Benutzergruppen ...............................68

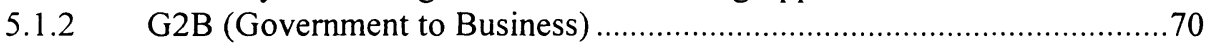

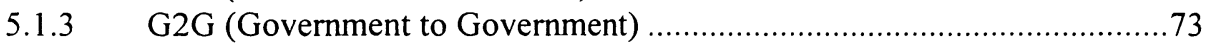

5.2 Beziehungskomplexität: Information, Kommunikation, Transaktion ........75

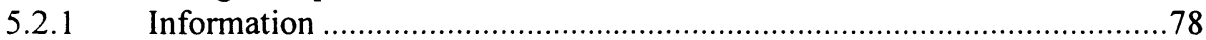

5.2.1.1 Information des Bürgers durch die Verwaltung ................................78

5.2.1.2 Komplexe Informationsdarbietungen ............................................8

5.2.1.3 Information der Verwaltung durch den Bürger .................................80

5.2.2 Kommunikation und allgemeine Dienstleistungen ..................................81

5.2.2.1 Verlagerungen zwischen Information und Kommunikation..................81

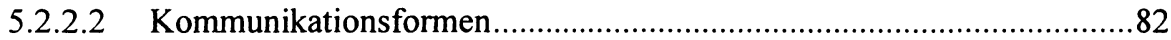

5.2.2.3 Heutiger Entwicklungsstand elektronischer G2C-Kommunikation........84

5.2.3 Transaktionen und spezielle Dienstleistungen .......................................86

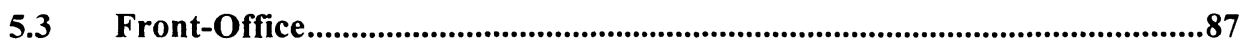

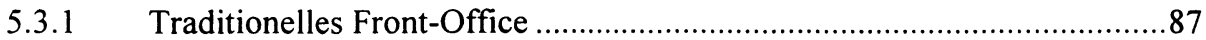

5.3.1.1 Schnittstelle zwischen Kunden und Verwaltung .................................87

5.3.1.2 Bürgeramt als organisatorisch integriertes Front-Office ......................91

5.3.2 Elektronisches Front-Office ..............................................................93

5.3.2.1 Elektronische Nachbildung bisheriger Front-Office-Funktionen ...........93

5.3.2.2 Übergang zum elektronischen Front-Office ........................................ 95 
5.3.2.3 Vorläufer des elektronischen Front-Office: BTX und Mailbox .............96

5.3.2.4 Elektronische Zugangswege zur Verwaltung ......................................98

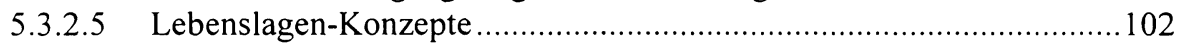

5.3.2.6 Organisationsformen des künftigen Front-Office .................................104

5.3.2.7 Anpassungszwang für das Back-Office ........................................... 106

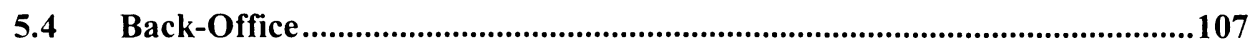

5.4.1 Back-Office als Produktionsstätte der Verwaltung ...............................107

5.4.2 Klassifizierungsansätze für das Back-Office......................................... 109

5.4.2.1 Das kundenbezogene Back-Office ................................................... 110

5.4.2.2 Querschnittsfunktionen im Back-Office ............................................ 113

5.4.2.3 Sonstige Funktionen des Back-Office ............................................. 116

5.4.3 Back-Office-Organisation und Produkte .............................................. 117

5.4.4 Heterogene Hardware-Landschaft.......................................................... 120

5.4.5 Manuelle Bearbeitungsverfahren ....................................................... 122

5.4.6 Rechnergestützte Fachverfahren ...................................................... 123

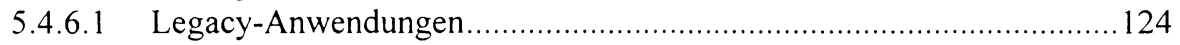

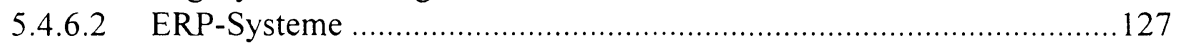

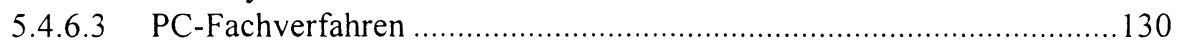

5.4.6.4 Anbindung an das Rechenzentrum ............................................... 131

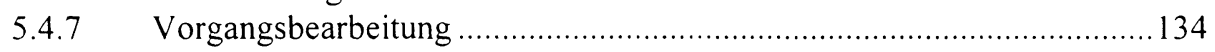

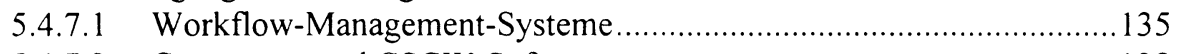

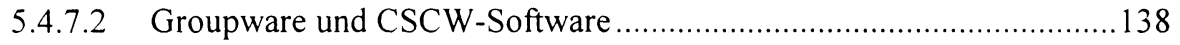

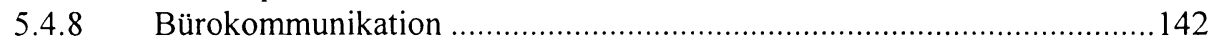

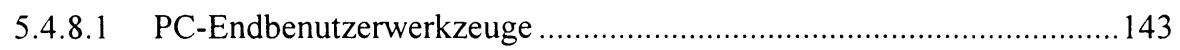

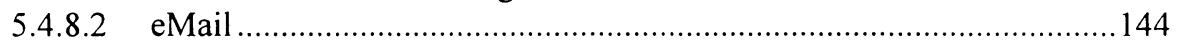

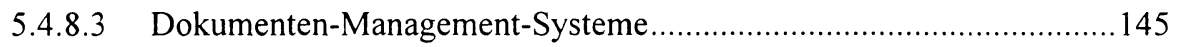

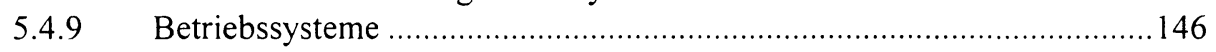

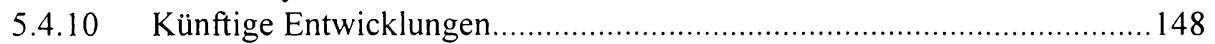

6. GRUNDLAGEN FORTGESCHRITTENEN EGOVERNMENTS............150

6.1 Standardisierung..........................................................................................151

6.1.1 Standardisierung und Standardisierungsinitiativen ...........................151

6.1.2 Standardisierung und Zweckbindung ............................................ 154

6.1.3 Standardisierung und das heutige Back-Office ................................... 156

6.1.4 Standardisierungsebenen und Lösungsansätze ................................... 159

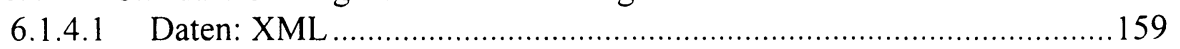

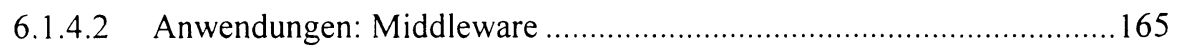

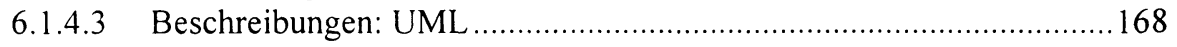

6.1.4.4 Geschäftsprozesse: Referenzmodelle ........................................... 169

6.1.4.5 Benutzeroberflächen: Web-Browser .................................................172

6.1.4.6 Dokumenten-, Ausweis- und Urkundenwesen................................... 172

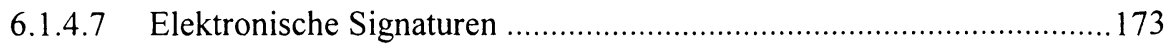

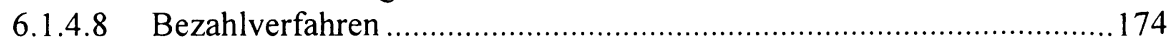

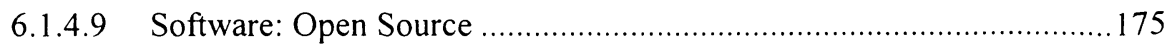

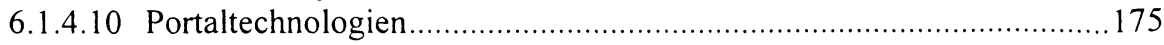


6.2 Integration im Back-Office ......................................................................177

6.2.1 Heterogenes Back-Office als Integrationsproblem..............................177

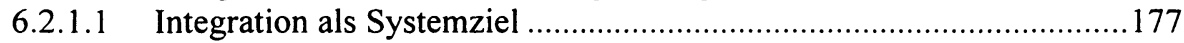

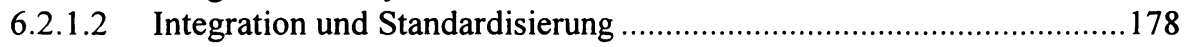

6.2.1.3 Integrationszwang im Back-Office................................................. 178

6.2.2 Perspektivenwechsel zur Prozeßintegration ......................................... 181

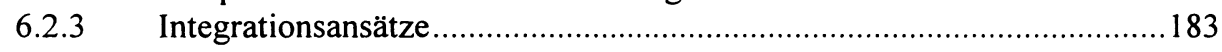

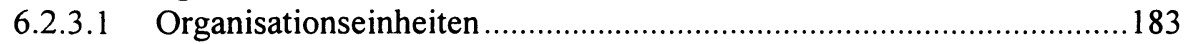

6.2.3.2 Externe Kooperationspartner und Mitarbeiter.................................... 184

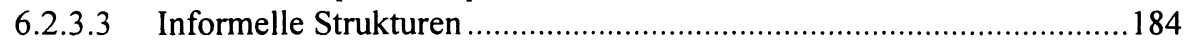

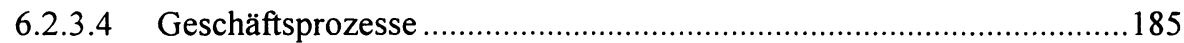

6.2.3.5 Daten- und Verfahrensintegration ............................................... 186

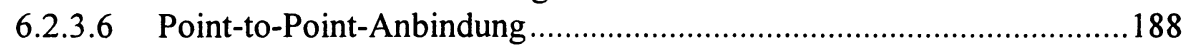

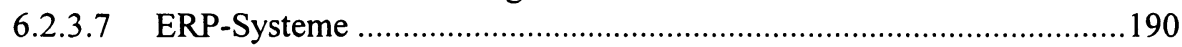

6.2.3.8 Middlewarelösungen: EAI und Web-Services ..................................191

6.3 eGovernment-Infrastrukturvoraussetzungen..........................................193

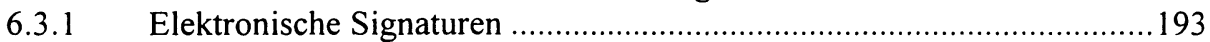

6.3.1.1 Formlose und formbedürftige Kommunikation .................................193

6.3.1.2 Eigenhändige Unterschrift und weitere Formerfordernisse ..................195

6.3.1.3 Anpassung rechtlicher Formvorschriften ......................................197

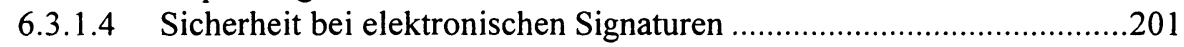

6.3.1.5 Funktionsweise digitaler Signaturen ................................................203

6.3.1.6 Private Key Verfahren ....................................................................204

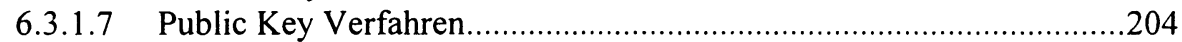

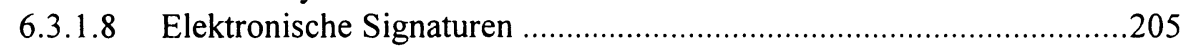

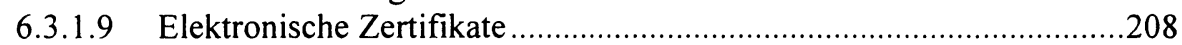

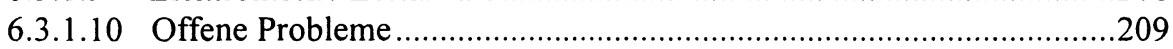

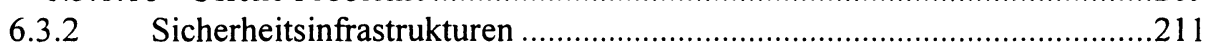

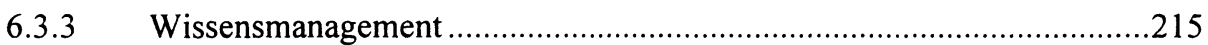

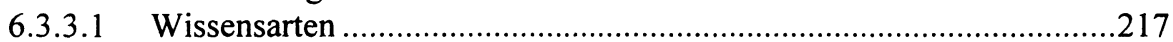

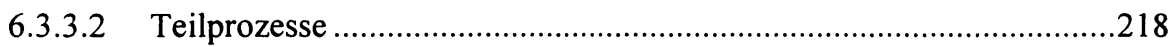

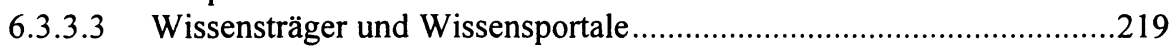

6.3.3.4 Softwareunterstützung für Wissensmanagementaufgaben....................220

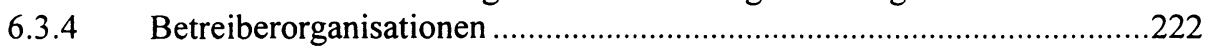

6.3.4.1 Neuartige Aufgaben im eGovernment-Umfeld ................................222

6.3.4.2 Künftige Betreiberorganisationen und Verfahrenshoheit ....................226

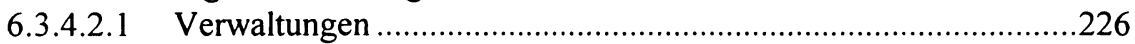

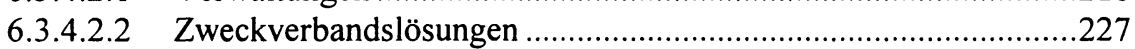

6.3.4.2.3 Public Private Partnerships ......................................................228

6.3.4.3 Strukturmerkmale der Betreiberaufgaben .........................................230

6.3.4.3.1 Strategische und operative Betreiberaufgaben ............................230

6.3.4.3.2 Prozeßbezug oder Querschnittsbezug .......................................232

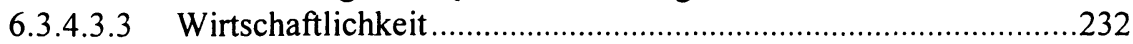

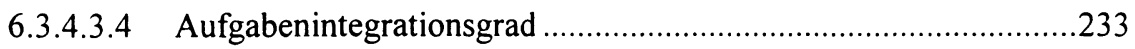

6.3.4.3.5 Back-Office-Betreiber und Front-Office-Betreiber .......................234 


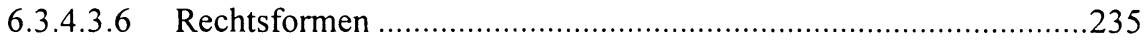

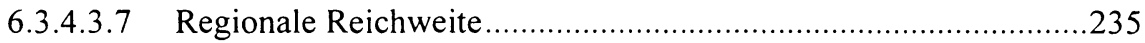

6.3.4.4 Aktuelle Schwerpunkte bei Betreiberaktivitäten .............................236

6.3.5 Elektronisches Ausweis-, Urkunden- und Dokumentenwesen .................238

6.3.5.1 Identitätsdokumente .................................................................240

6.3.5.2 Sonstige Ausweispapiere, Dokumente, Bescheinigungen ...................240

6.3.5.3 Grenzen elektronischer Dokumentenführung ....................................242

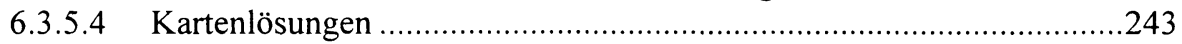

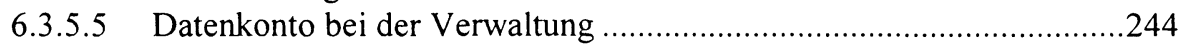

6.3.5.6 Datenkonto beim Bürger ..................................................................245

6.3.5.7 Virtuelle Dokumente als Ausweisäquivalent....................................2.245

6.3.5.8 Karten- versus serverbasierte Lösungen............................................246

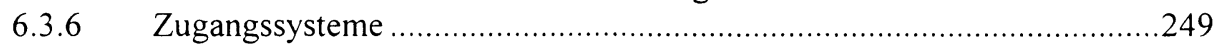

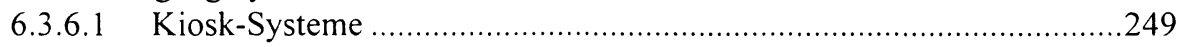

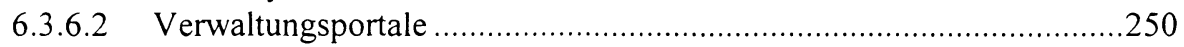

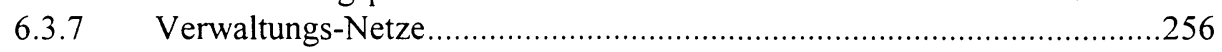

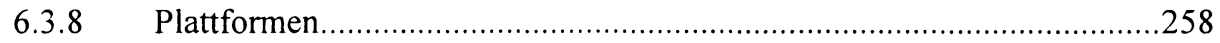

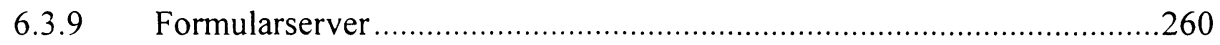

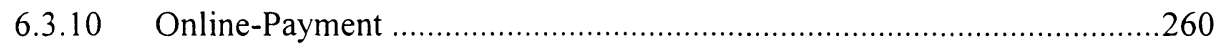

7. eGOVERNMENT-SCHWERPUNKTE UND PROJEKTBEISPIELE ....263

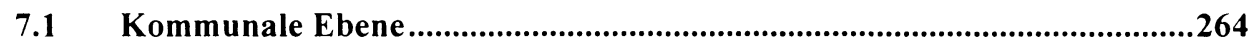

7.2 Landes-, Bundes- und internationale Entwicklungen ..............................270

7.3 Exemplarische Lösungsansätze....................................................................273

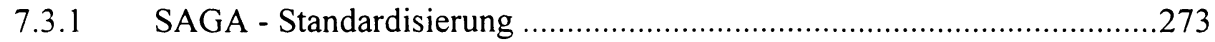

7.3.2 OSCI und Governikus - Standardisierungsansatz und Lösungsplattform. 273

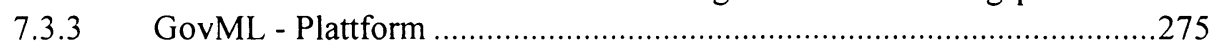

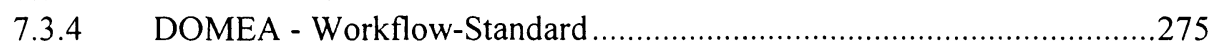

7.3.5 ELSTER - Elektronische Steuererklärung ...........................................276

7.3.6 SPHINX - Sichere eMail-Kommunikation ..........................................277

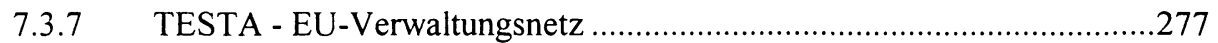

7.3.8 Akteneinsicht und Informationsrechte ...............................................278

7.3.9 Bundeseinheitliche Wirtschaftsnummer............................................279

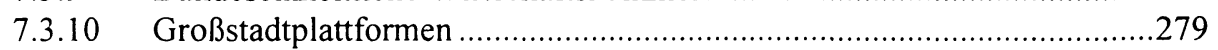

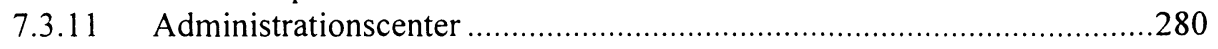

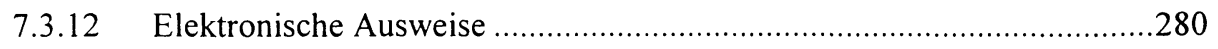

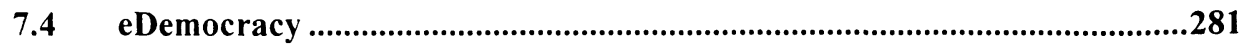

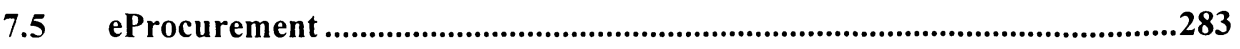

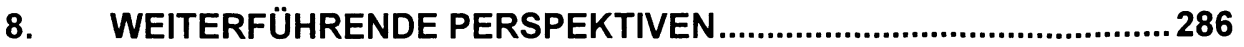




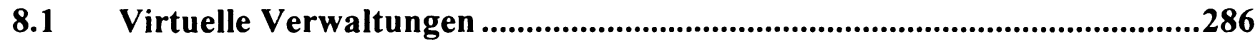

8.2 Verhältnis zwischen traditioneller Verwaltung und eGovernment-Betreibern............................................................................290

8.3 Messung des Entwicklungsstandes beim eGovernment..............................294

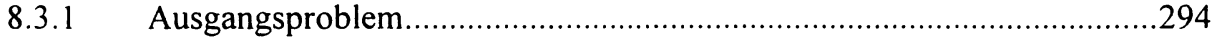

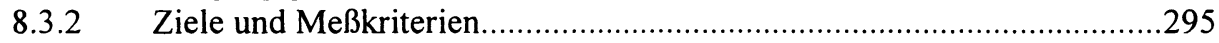

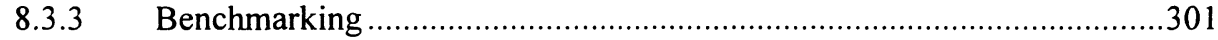

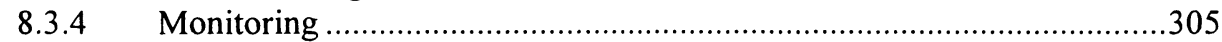

8.4 Administrative Einstiegswege ins eGovernment ....................................306

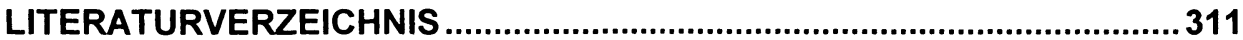

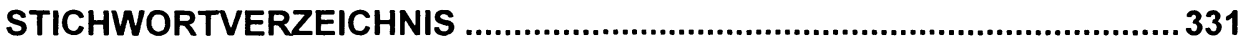




\section{ABBILDUNGSVERZEICHNIS}

Abbildung 1: Von der ADV zum eGovernment.................................................

Abbildung 2: Entwicklungsschritte zur Online-Verwaltung ................................... 13

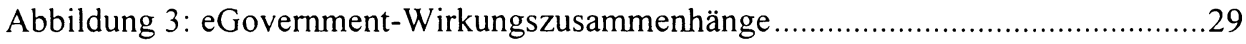

Abbildung 4: Voraussetzungsschwellen am Beispiel der elektronischen Signatur ........31

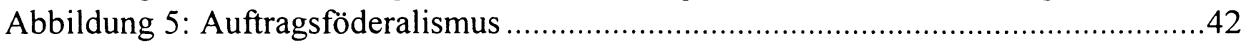

Abbildung 6: Föderalistische Perspektiven im Internet ............................................44

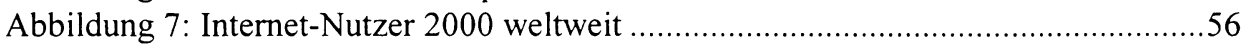

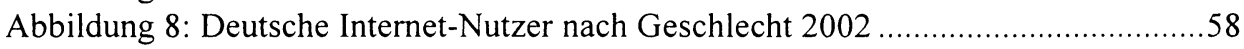

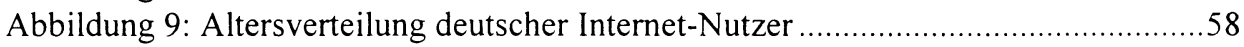

Abbildung 10: Berufsausbildung deutscher Internet-Nutzer .................................59

Abbildung 11: G2C/G2E-Beziehung: interne und externe Verwaltungskunden ...........66

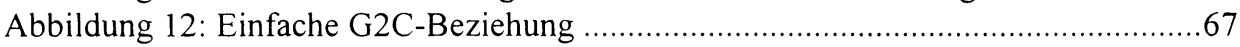

Abbildung 13: n: 1-Beziehung zwischen Verwaltung und Bürgern .............................68

Abbildung 14: Übergang zwischen Information, Kommunikation, Transaktion............75

Abbildung 15: Einfache Informationsanfrage ........................................................ 78

Abbildung 16: Einfache Kommunikation zwischen Bürger und Verwaltung ................81

Abbildung 17: Online-Diskussionsforum zwischen Bürger und Verwaltung.................81

Abbildung 18: Neuartige Kommunikation zwischen Bürgern und Verwaltung .............83

Abbildung 19: PDF-Formulare als erster Schritt ins eGovernment ...............................85

Abbildung 20: Segmentiertes Front-Office in der Offline-Verwaltung........................89

Abbildung 21: Modernisiertes Back-Office mit Bürgerämtern ....................................92

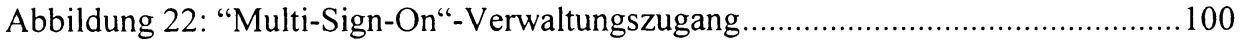

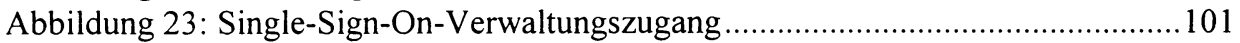

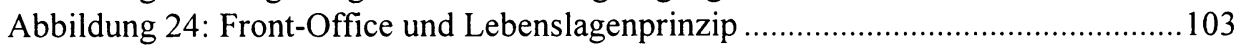

Abbildung 25: Multi-Channel-Zugang beim künftigen Front-Office ........................... 105

Abbildung 26: Back-Office-Segmente ............................................................. 110

Abbildung 27: Einfaches Beispiel für ein publikumsnahes Back-Office .....................111

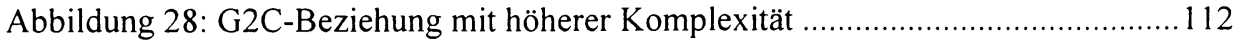

Abbildung 29: Beispiel Produktbereich Verkehrswesen.......................................... 119

Abbildung 30: Manueller Workflow bei Reisekosten ........................................... 135

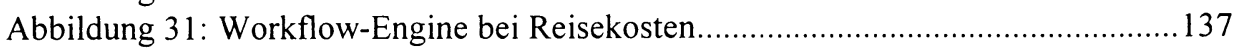

Abbildung 32: Kommunikationsintensität bei Gruppenarbeit..................................139

Abbildung 33: Client-Server-Systeme in der Verwaltung.........................................148

Abbildung 34: Objektorientiertes Referenzschema der Vorgangsbearbeitung ............ 169

Abbildung 35: Point-to-Point-Anwendungsintegration........................................... 189

Abbildung 36: Anwendungsintegration mit Kommunikationsserver ......................... 190

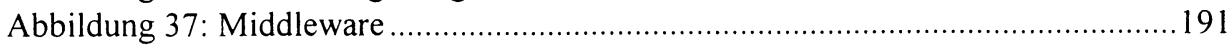

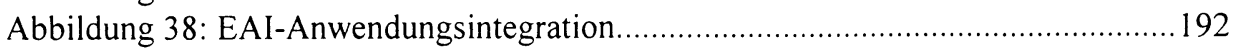

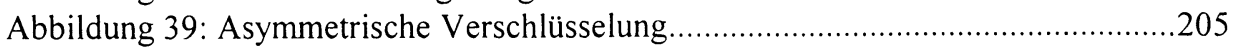

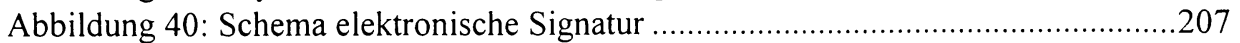

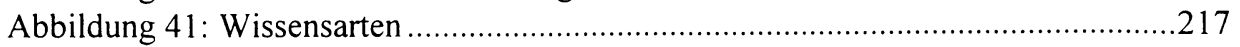

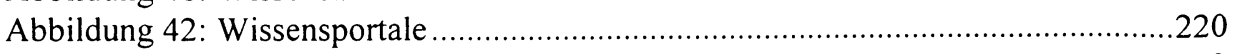

Abbildung 43: Betreiberorganisationen im Aufbau .............................................223 


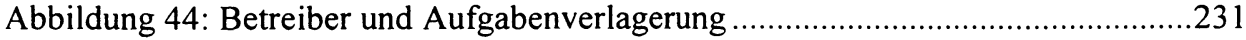

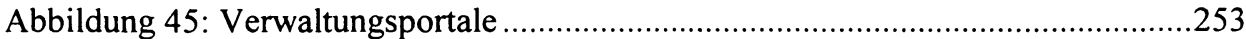

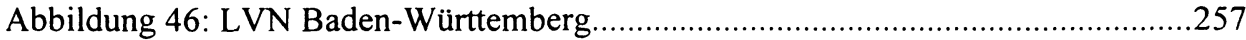

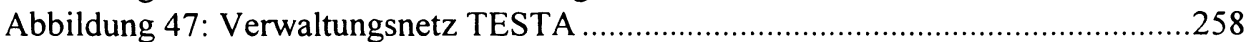

Abbildung 48: eGovernment: von Offline- zur Online-Betreibern ............................292 


\section{TABELLENVERZEICHNIS}

Tabelle 1: Beispiel: Fünf-Schichten-Modell für eGovernment ..................................26

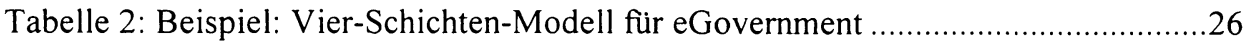

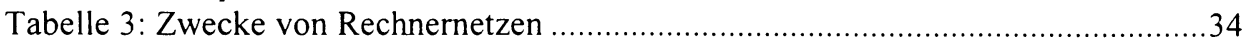

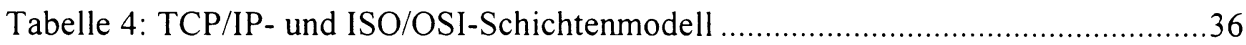

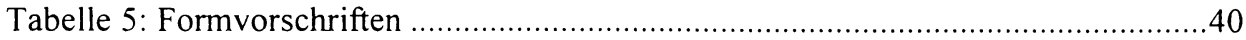

Tabelle 6: Online-Nutzer nach Land, Haushaltsanteil und monatlicher Onlinezeit .......57

Tabelle 7: Kundenbeziehungen der Verwaltung im eGovernment ............................63

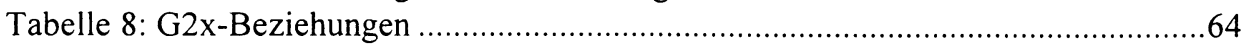

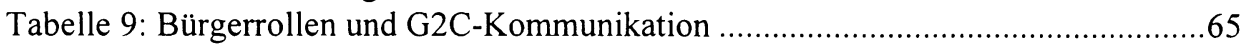

Tabelle 10: G2B-Kernaktivitäten....................................................................... 71

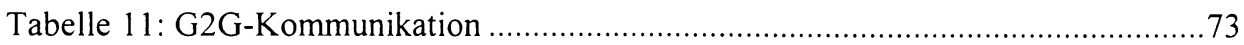

Tabelle 12: Verbindlichkeitsgrad und technischer Aufwand bei Kommunikationen .....77

Tabelle 13: Exemplarische Angebote eines städtischen Informationssystems ...............79

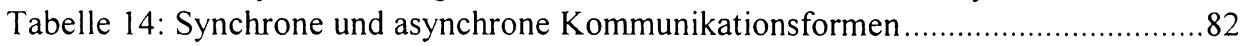

Tabelle 15: Anwendungsfunktionen des Front-Office .......................................... 91

Tabelle 16: Funktionen des Front-Office: kurz- bis mittelfristige Umsetzbarkeit..........94

Tabelle 17: Funktionen des Front-Office: längerfristige Umsetzbarkeit .......................95

Tabelle 18: Front-Office zwischen Verwaltung und Kunden......................................96

Tabelle 19: Kundenbezogenes Back-Office in der Kommunalverwaltung ..................111

Tabelle 20: Querschnittsbereiche des Back-Office ................................................ 114

Tabelle 21: Back-Office: Datenaustausch mit anderen Behörden...............................116

Tabelle 22: Kommunaler Produktplan Baden-Württemberg .....................................118

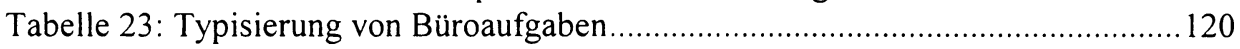

Tabelle 24: zentrale RZ-Systeme für Stapel- und Dialogverarbeitung.......................121

Tabelle 25: dezentrale Systeme bei Mitgliedern und Kunden...................................122

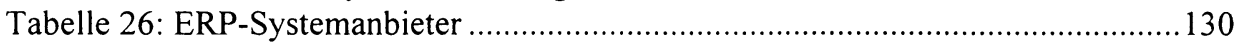

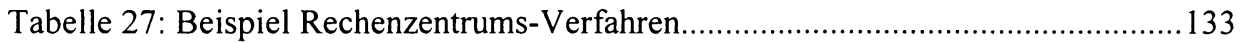

Tabelle 28: Information, Koordination, Kollaboration, Kooperation ...........................139

Tabelle 29: Workflow und Groupware als Elemente der Vorgangsbearbeitung........... 140

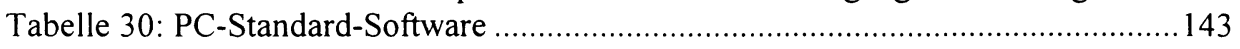

Tabelle 31: Systeme für Großrechner und mittlere Datentechnik ............................ 146

Tabelle 32: Nationale und internationale Standardisierungsorganisationen ................ 153

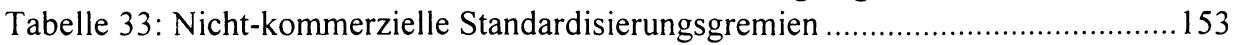

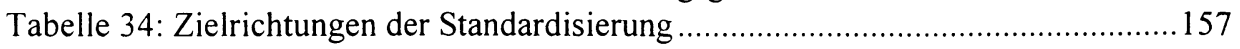

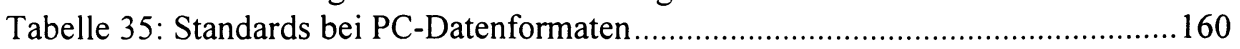

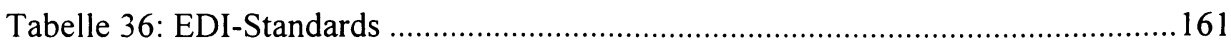

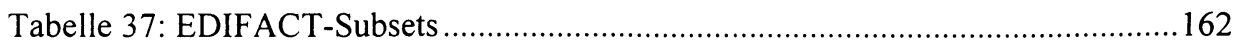

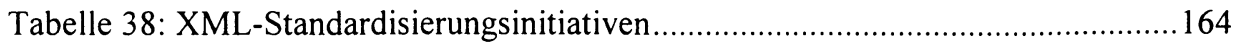

Tabelle 39: Middleware: Schicht zwischen Betriebssystem und Anwendung .............. 166

Tabelle 40: Schriftformfunktionen in elektronischen Verwaltungsprozessen ...............196

Tabelle 41: Gesetzliche Schriftform im $\S 126$ BGB und Novellierung ........................198

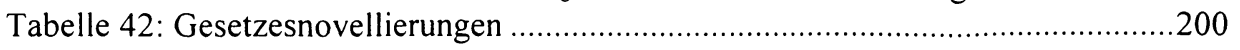

Tabelle 43: Sicherheitsstufen bei Text- und elektronischer Form..............................202 
Tabelle 44: Sicherheit bei Brief, eMail und signierter eMail..................................202

Tabelle 45: Einfache, qualifizierte und akkreditierte Signaturverfahren......................203

Tabelle 46: Ablaufschema elektronische Signatur ..................................................208

Tabelle 47: Aufbau einer dreistufigen Sicherheitsinfrastruktur ...............................213

Tabelle 48: Akkreditierte Zertifizierungsdiensteanbieter.........................................214

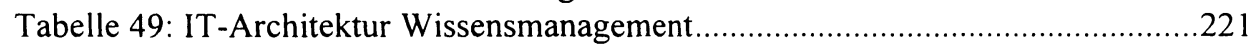

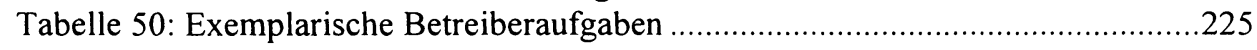

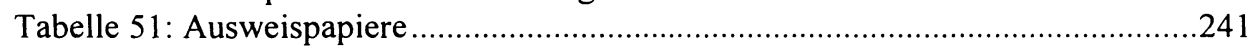

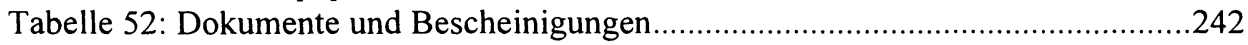

Tabelle 53: eGovernment-Schwerpunkte in Großstädten 2002 ................................266

Tabelle 54: Kommunale Web-Sites nach Gemeindegrößenklassen ..........................267

Tabelle 55: Aktualisierungsraten bei Web-Sites .....................................................267

Tabelle 56: Hauptamtliche Web-Site-Betreuer nach Gemeindegrößenklassen.............268

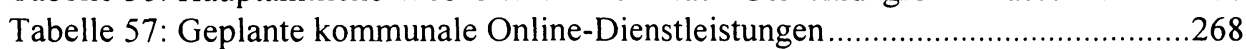

Tabelle 58: Kommunale Online-Dienstleistungen in Baden-Württemberg.................269

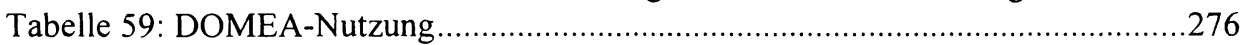

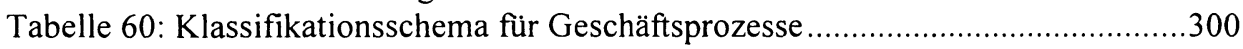

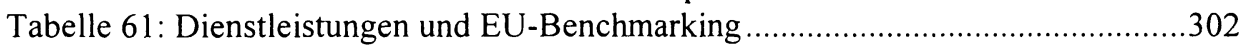




\section{ABKÜRZUNGSVERZEICHNIS}

\begin{tabular}{|c|c|}
\hline AIG & Akteneinsichts- und Informationszugangsgesetz \\
\hline $\mathrm{B} 2 \mathrm{~B}$ & Business-to-Business \\
\hline $\mathrm{B} 2 \mathrm{C}$ & Business-to-Customer \\
\hline $\mathrm{B} 2 \mathrm{~N}$ & Business-to-NGO \\
\hline BAFI & Bundesamt für die Anerkennung ausländischer Flüchtlinge \\
\hline BAköV & Bundesakademie für öffentliche Verwaltung \\
\hline BDSG & Bundesdatenschutzgesetz \\
\hline BEGIX & Balanced-E-Government-IndeX \\
\hline BMI & Bundesministerium des Innern \\
\hline $\mathrm{BMJ}$ & Bundesministerium der Justiz \\
\hline BMWi & Bundesministerium für Wirtschaft \\
\hline bps & Bits pro Sekunde \\
\hline BPS & Bytes pro Sekunde \\
\hline BSI & Bundesamt für Sicherheit in der Informationstechnik \\
\hline BTX & Bildschirmtext \\
\hline CASE & Computer Aided Software Engineering \\
\hline $\mathrm{CIO}$ & Chief Information Officer \\
\hline CLI & Command Line Interface \\
\hline CMS & Content-Management-Systeme \\
\hline CORBA & Common Object Request Broker Architecture \\
\hline CRM & Customer-Relationship-Management \\
\hline $\mathrm{C} / \mathrm{S}$ & Client/Server \\
\hline $\mathrm{CSCW}$ & Computer Supported Cooperative Work \\
\hline DIGANT & digitales Antragsverfahren für Reisepässe und Personalausweise \\
\hline DMS & Dokumenten-Management-System \\
\hline DRT & Document Related Technologies \\
\hline DTD & Data Type Definition \\
\hline EAI & Enterprise Application Integration \\
\hline EDI & Electronic Data Interchange \\
\hline EDIFACT & $\begin{array}{l}\text { Electronic Data Interchange for Administration, Commerce and Trans- } \\
\text { port }\end{array}$ \\
\hline EESSI & European Electronic Signature Standardization Initiative \\
\hline ELSTER & Elektronische Steuererklärung \\
\hline ERP & Enterprise Resource Planning \\
\hline FASME & Facilitating Administrative Services for Mobile Europeans \\
\hline FDDI & fiber distributed data interface \\
\hline $\mathrm{G} 2 \mathrm{E}$ & Government-to-Employee \\
\hline $\mathrm{G} 2 \mathrm{G}$ & Government-to-Government \\
\hline $\mathrm{G} 2 \mathrm{C}$ & Government-to-Customer \\
\hline G2B & Government-to-Business \\
\hline $\mathrm{G} 2 \mathrm{~N}$ & Government -to-NGO \\
\hline GI & Gesellschaft für Informatik e.V. \\
\hline GUI & Graphical user interface \\
\hline
\end{tabular}




\begin{tabular}{|c|c|}
\hline HKR & Haushalts-, Kassen- und Rechnungswesen \\
\hline HTML & Hyper Text Markup Language \\
\hline IDA & Interchange of Data between Administrations \\
\hline IDL & Interface Definition Language \\
\hline IFA & Internationale Funkausstellung \\
\hline ISO & International Organization for Standardization \\
\hline ITG & Informationstechnische Gesellschaft \\
\hline IVBB & Informationsverbund Berlin-Bonn \\
\hline $\mathrm{J} 2 \mathrm{EE}$ & Java 2 Enterprise Edition \\
\hline KBA & Kraftfahrtbundesamt \\
\hline KBSt & $\begin{array}{l}\text { Koordinierungs- und Beratungsstelle der Bundesregierung für } \\
\text { Informationstechnik in der Bundesverwaltung }\end{array}$ \\
\hline KGSt & Kommunale Gemeinschaftsstelle für Verwaltungsvereinfachung \\
\hline $\mathrm{KI}$ & Künstliche Intelligenz \\
\hline KM & Knowledge Management \\
\hline KoopA ADV & Kooperationsausschuß ADV Bund, Länder, Kommunaler Bereich \\
\hline LAN & Local Area Network \\
\hline LDAP & Lightweight Directory Access Protocol \\
\hline LVN & Landesverwaltungsnetz \\
\hline MDA & Model Driven Architecture \\
\hline MDT & Mittlere Datentechnik \\
\hline MIAS & Mehrwertsteuer-Informationsaustauschsystem \\
\hline $\mathrm{NGO}$ & Non-Governmental-Organizations \\
\hline NPM & New Public Management \\
\hline NSM & Neues Steuerungsmodell \\
\hline $\mathrm{OAG}$ & Open Applications Group \\
\hline OCF & Open Card Framework \\
\hline OCR & Optical Character Recognition \\
\hline OMG & Object Management Group \\
\hline ORB & Object Request Broker \\
\hline OSCAR & Online Services Computer Interface Architecture \\
\hline OSCI & Online Services Computer Interface \\
\hline OSS & Open Source Software \\
\hline PDF & Portable Document Format \\
\hline PIN & Personen Identifikations-Nummer \\
\hline PK & Personenkennzeichen \\
\hline PKI & Public Key Infrastructure \\
\hline PPP & Public Private Partnership \\
\hline RegTP & Regulierungsbehörde für Telekommunikation und Post \\
\hline SGML & Structured Generalized Markup Language \\
\hline SAGA & Standards und Architekturen für eGovernment Anwendungen \\
\hline SigG & Signaturgesetz \\
\hline SET & Secure electronic transaction \\
\hline SMS & Short Message Service \\
\hline SNA & Systems Network Architecture \\
\hline SOAP & Simple Object Access Protocol \\
\hline
\end{tabular}




$\begin{array}{ll}\text { TCP/IP } & \text { Transmission Control Protocol / Internet Protocol } \\ \text { TESTA } & \text { Trans-European Services for Telematics between Administrations } \\ \text { UDDI } & \text { Universal Description, Discovery and Integration } \\ \text { UML } & \text { Unified Modeling Language } \\ \text { UMTS } & \text { Universal Mobile Telecommunication Standard } \\ \text { URL } & \text { Uniform Resource Locator } \\ \text { VAN } & \text { Value Added Network } \\ \text { VPN } & \text { Virtuelles privates Netzwerk } \\ \text { W3C } & \text { World Wide Web Consortium } \\ \text { WAN } & \text { Wide Area Network } \\ \text { WMS } & \text { Workflow-Management-System } \\ \text { WORM } & \text { Write Once, Read Many } \\ \text { WWW } & \text { World Wide Web } \\ \text { WSDL } & \text { Web Services Description Language } \\ \text { XML } & \text { Extensible Markup Language }\end{array}$

